\title{
Desvanecimiento de la frontera como límite. Imaginario del borde como espacio público físico y virtual
}

\author{
Fading borders as limits. Imaginary of borders as a physical and virtual public space \\ Desaparecimento da fronteira como limite. Imaginário da borda como espaço público físico e virtual
}

\begin{abstract}
Gabriela Eloísa Muñoz-Torres Susana Gutiérrez-Luna

Universidad de Guadalajara, Guadalajara (México)

Centro Universitario de Arte, Arquitectura y Diseño
\end{abstract}

Muñoz-Torres, G., \& Gutiérrez-Luna, S. (2019). Desvanecimiento de la frontera como límite. Imaginario del borde como espacio público físico y virtual. Revista de Arquitectura (Bogotá), 21(2), 33-43. doi:http://doi. org/10.14718/RevArq.2019.21.2.2133

\author{
Gabriela Eloísa Muñoz-Torres \\ Arquitecta, Universidad Autónoma de Ciudad Juárez, Ciudad Juárez \\ (México). \\ Maestría en Procesos y Expresión Gráfica en la Expresión Arquitectó- \\ nica y Urbana, Universidad de Guadalajara (México). \\ Candidata a Doctora, doctorado Ciudad, Territorio y Sustentabilidad, \\ Universidad de Guadalajara (México) \\ (D) https://orcid.org/0000-0002-1905-7109 \\ gabriela.munoz@edu.uag.mx \\ Susana Gutiérrez-Luna \\ Arquitecta, Universidad Autónoma de Ciudad Juárez, Ciudad Juárez \\ (México). \\ Maestría en Procesos y Expresión Gráfica en la Expresión Arquitectó- \\ nica y Urbana, Universidad de Guadalajara (México). \\ Candidata a Doctora. Doctorado Ciudad, Territorio y Sustentabilidad, \\ Universidad de Guadalajara (México) \\ (i) https://orcid.org/0000-0003-3282-1914 \\ susanag.luna@gmail.com
}

\section{Resumen}

Por lo general, las fronteras se entienden como límites y divisiones con diferenciaciones físicas y culturales marcadas, es el caso de fronteras geográficas entre dos países en condiciones tan dispares como México y Estados Unidos, a tal punto que se plantea la necesidad de erigir muros que separen. Por esta razón, surge la inquietud de conocer cuáles son las prácticas y los imaginarios presentes en los habitantes respecto a su situación como fronterizos y a la idea de fractura urbana. Así, en esta investigación se propone el análisis del imaginario alrededor del borde fronterizo en tanto un lugar que puede unir o separar. El enfoque metodológico se basa en entrevistas, encuestas, análisis de registros mediáticos de actividades de integración y registros fotográficos presentes en medios de comunicación. En los resultados se expone que el imaginario presente en la frontera entre Ciudad Juárez (México) y EI Paso (EE.UU.) permite las expresiones físicas y virtuales de los habitantes y tiende a desvanecer los límites, lo que convierte el borde fronterizo en un espacio público natural y artificial, consolidado y propicio para la manifestación de una identidad compartida.

Palabras clave: ciudadanía; espacios de encuentro; espacios transnacionales; frontera México-Estados Unidos; identidad cultural; imaginarios urbanos; representaciones sociales.

\section{Abstract}

In general, borders are understood as limits and divisions with marked physical and cultural differentiations, as is the case of geographical borders between two countries with drastically different conditions like Mexico and the United States, such that the need to erect walls to separate them is proposed. For this reason, the question of what practices and imaginaries are present in residents regarding their situation in the border and the idea of urban fracture is relevant. Thus, this research proposes the analysis of the imaginary regarding the border as a place that can unite or separate. The methodological approach is based on interviews, surveys, analysis of media records of integration activities, and photographic records in media outlets. The results present that the imaginary present along the border between Ciudad Juarez (Mexico) and EI Paso (United States) allows the physical and virtual expressions of residents and tends to fade limits, which converts the border zone into a consolidated natural and artificial public space, conducive to the manifestation of a shared identity.

Keywords: Citizenry, meeting spaces, transnational spaces, U.S.-Mexico border, cultural identity, urban imaginaries, social representations.

\section{Resumo}

Em geral, as fronteiras são entendidas como limites e divisões com diferenças físicas e culturais marcadas. É o caso de fronteiras geográficas entre dois países em condições tão desiguais como o México e os Estados Unidos, a tal ponto que é planejado levantar muros que os separem. Por isso, surge a inquietação de conhecer quais são as práticas e os imaginários presentes nos habitantes a respeito de sua situação como fronteiriços e da ideia de fratura urbana. Assim, nesta pesquisa, é proposta a análise do imaginário acerca da borda fronteiriça como um lugar que pode unir ou separar. A abordagem metodológica está baseada em entrevistas, enquetes, análise de registros midiáticos de atividades de integração e registros fotográficos de meios de comunicação. Nos resultados, é exposto que o imaginário presente na fronteira entre Ciudad Juárez (México) e El Paso (Estados Unidos) permite as expressões físicas e virtuais dos habitantes e tende a desaparecer os limites, o que converte a borda fronteiriça em um espaço público natural e artificial, consolidado e propício à manifestação de uma identidade partilhada.

Palavras-chave: cidadania; espaços de encontro; espaços transnacionais; fronteira MéxicoEstados Unidos; identidade cultural; imaginários urbanos; representações sociais. Evaluado: abril $10 / 2019$

\section{Introducción}

Esta investigación fue desarrollada en el marco del programa académico de doctorado Ciudad Territorio y Sustentabilidad, de la Universidad de Guadalajara, México, con el apoyo económico del Consejo Nacional de Ciencia y Tecnología (Conacyt), a través del financiamiento que otorga para la formación de capital humano, científico y tecnológico. El objetivo del presente trabajo es identificar los imaginarios compartidos que se expresan en las dinámicas sociales y prácticas habituales de los habitantes de las ciudades fronterizas del norte de México, respecto al borde fronterizo como espacio público (físico y virtual).

La hipótesis parte de afirmar que los habitantes, lejos de replicar dinámicas sociales que refuerzan la idea de la frontera como fragmentación y segregación, tienden a desvanecer los límites impuestos por la delimitación transnacional y así, en lo colectivo de los imaginarios y sus prácticas, conforman un espacio unificado y compartido.

El concepto de frontera se entiende como "el espacio fisco y social existente entre dos países, lo cual hace de la frontera un lugar único y extraordinario" (Pineda y Herrera, 2007, p. 10). Las fronteras a lo largo del territorio mexicano poseen características socioespaciales tan particulares, que las convierten en puntos estratégicos y de gran relevancia para la formulación de análisis desde múltiples disciplinas, focalizados en tópicos a razón del interés que despiertan sus dinámicas económicas, los intercambios migratorios, la agenda política, los impactos ambientales, la gestión del suelo, las prácticas culturales o las imbricadas cargas simbólicas que se gestan desde y para la sociedad. 
Ya sea que se trate de la frontera sur en colindancia con Guatemala y Belice, o la frontera norte en colindancia con Estados Unidos de América, ambas fronteras representan espacios de hibridación que, aunque contextualizadas en extremos opuestos literal y figurativamente, comparten ciertos elementos comunes que las identifican como territorios en conflicto por el límite, por la permanencia de lo transitorio, la resistencia ante los grupos de delincuencia organizada y el contrabando, e incluso el estigma de la violencia, el narcotráfico y una identidad fluctuante que, como su población, se encuentra inacabada.

Aunque es usual entender la frontera en su aspecto físico-territorial, es de resaltar que en su origen estas se gestaron como fronteras culturales establecidas en función de aspectos compartidos socialmente, de índole simbólica, identitaria y cultural (Brenna, 2011). También es común identificar el concepto de frontera en términos de ruptura, separación o incluso como obstáculo, sin embargo, la realidad es que esta representa dualidades indisociables. Con ello los habitantes fronterizos construyen representaciones de la ciudad en función de la complejidad que significa tal dualidad, pues mientras que la frontera es el espacio de la división, del encuentro, y a la vez que es la manifestación del enfrentamiento con el otro, es la materialización del diálogo cultural; recientemente, también se puede considerar el espacio de tensión entre la realidad física y una realidad digital, habilitada por las omnipresentes redes informáticas, que ha adquirido algunas de las atribuciones del lugar físico, como escenario en transición donde los valores simbólicos mediados por información digital, más que los valores inherentes de las cosas, son los que conducen la producción y la transformación cultural hacia el flujo constante de una realidad dinámica (Ito, 2006, p. 20).

Se reconoce como un proceso que no es uniforme ni equitativo, que incide irreversiblemente en la relación con los recursos naturales, en los límites del concepto de territorialidad y en la modificación de prácticas e identidades locales. La confrontación de modelos culturales sugiere ajustar el paradigma para entender la ciudad contemporánea vinculándola a los procesos y patrones que dan cohesión a las transformaciones del estadio tecnológico de un asentamiento específico. En este sentido, cabe destacar el marcado contraste presente en las ciudades fronterizas (Morales y Cabrera, 2016)

La construcción de la ciudad anclada en la frontera es la propia construcción del espacio público. Fernando Carrión señala que "el espacio público es la esencia de la ciudad [...] es la ciudad misma [...]. El espacio público no existe si no es en relación con la ciudad" (2016, p. 23), con lo que se entiende que el espacio público es sinónimo de ciudad, espacio donde se legitiman los valores, anhelos e ideales compartidos socialmente por sus habitantes.

Carrión (2016) también pone de manifiesto el debate sobre la capacidad del espacio público contemporáneo como contenedor del intercambio y el encuentro con el otro, debido a fenómenos como la globalización, la revolución científico-tecnológica y la transición a los espacios de los flujos, conceptualizados por Castells (2006), que resultan en la domiciliación de diferentes actividades (trabajo, estudio y ocio on line) y la simultánea expulsión del espacio público por miedo, inseguridad y violencia. En el contexto de lo que Carrión enmarca como el nuevo urbanismo dentro de la realidad latinoamericana, "la ciudad pierde las posibilidades de construcción y de cohesión sociales, se reduce la participación, se restringe la ciudadanía y se ausenta el estímulo a las prácticas de tolerancia" (2016, p. 34). Entonces ¿cuál es el futuro del espacio público que define la ciudad en el contexto tecnológico en la frontera? ¿Cómo se reconfigura el imaginario de este espacio público fronterizo? García Canclini (2000, p. 17) abona al debate el planteamiento de que es "en los medios masivos de comunicación donde se desenvuelve para la población el espacio público". Así, resulta pertinente indagar sobre el impacto que el desarrollo tecnológico tiene en las dinámicas que cuestionan y acotan la concepción del límite en los imaginarios del espacio público fronterizo.

\section{Los imaginarios}

Después del fracaso del enfoque netamente positivista para hacer referencia a la realidad que define el fenómeno urbano, resurge con fuerza la valoración de los enfoques hermenéuticos, que rescatan y reconocen la importancia de las subjetividades y el peso de lo simbólico. Los imaginarios, en tanto colectivos son "esquemas, construidos socialmente, que nos permiten percibir algo como real, explicarlo e intervenir operativamente en lo que en cada sistema social se considere como realidad" (Pintos, 1994, pp. 571-572).

En primera instancia, los imaginarios resultan en complejos procesos para su identificación, análisis y posterior tratamiento, que dificultan incluso el establecimiento de una conceptualización única y globalmente aceptada, pues al ser el entramado de subjetividades sociales su materia prima, se presta a diferentes abordajes. Castoriadis, en tanto uno de los autores indispensables en el estudio de los imaginarios, menciona que: "Lo imaginario del que hablo no es imagen de. Es creación incesante y esencialmente indeterminada (social-histórico y psíquico) de 
figuras/formas/imágenes, a partir de las cuales solamente puede tratarse de 'alguna cosa'. Lo que llamamos realidad y racionalidad son obras de ello" (1975, p. 5).

Y continúa reforzando en el mismo texto la idea de la generación de los imaginarios irremediablemente con la participación de el otro, es decir, el imaginario participa de la negociación narrativa y discursiva de la realidad percibida e imaginada de la otredad, que mediante su repetición, ampliación, transformación y eventual estabilización, se institucionaliza y asume de manera consciente e inconsciente. Por otro lado, Armando Silva (2001b, p. 108) establece que los imaginarios "apuntan a una categoría cognitiva para referirnos a la experiencia humana de construir percepciones desde donde somos sociales, no por conveniencias sino por deseos, anhelos o frustraciones".

Con ello se puede establecer que la importancia del análisis de estudio de los imaginarios, en este caso del espacio público fronterizo, se constituye como una pieza clave para la interpretación y la reflexividad ${ }^{1}$, necesaria para la integración de la sociedad contemporánea. Así, la imbricación de procesos subjetivos y sumamente significativos en la sociedad, como las creencias, las identidades, las imágenes y los procesos colectivos, a diferencia de las representaciones sociales, también implican el intercambio y la identificación de lo aspiracional e imaginable, legitimado en masa para su reconocimiento como imaginario dominante, que inevitablemente deriva en la proyección del espacio público fronterizo en el futuro. ¿Cómo es ese espacio público fronterizo proyectado? ¿Qué es capaz de imaginar el habitante fronterizo? ¿Qué recibe del entorno que nutre sus propias evocaciones?

\section{Imaginarios urbanos}

Una vez concebidos, los imaginarios han encontrado campo fértil desde diferentes líneas de investigación. Coca, Valero, Randazzo y Pintos (2015) enumeran cinco principales: sociología teórica, sociología urbana, sociología de la ciencia y la tecnología, teoría sociológica y análisis histórico. En este sentido, al optar por la línea de la sociología urbana, se retoman la conceptualización y los trabajos del imaginario abordados desde y para la ciudad, en donde se define que "el espacio urbano, no es solo una entidad física, sino que es también un territorio imaginado por sus habitantes" (p. 114).

Por su parte, en una aproximación a los imaginarios urbanos, Silva (2001b) afirma que, en la configuración de la imagen de la ciudad entre

1 Acto imprescindible desde el enfoque del fenómeno de la modernidad reflexiva. los ciudadanos, se alude a la integración de una ciudad subjetiva que, a través de la comunicación de los sujetos, es vivida, interiorizada, proyectada y recorrida, que por fuerza escapa del territorio físico hacia lo que denomina una utopía y, por tanto, una ensoñación.

Paralelamente, en función del corte tecnológico que atiende este trabajo, Silva (2001a) ofrece argumentos sobre la transición que experimentan las ciudades, y, por ende, el espacio públi$\mathrm{Co}$, en el que ocurre el traslape de lo físico, lo abstracto, lo figurativo y lo imaginario, ahora en transición hacia lo mediático-digital -en deuda con los experimentos de representación de las vanguardias y con implicaciones en la teoría y la producción del espacio-:

\footnotetext{
Algunos recientes filmes muestran la analogía entre el mundo virtual urbano construido por las computadoras y los espacios de una ciudad donde lo propiamente físico y palpable es vivido desde la clonación tecnológica. Hoy, pues, cuando la ciudad en su avance desterritorializador, en ese ocaso señalado por varios estudiosos, donde las fronteras que "parecían acotarla como espacio definido y que en su condición de tal demarcaban el afuera del adentro", llega al punto en el que lo urbano les impone desde afuera, para acabar siendo ella un espacio sin fronteras. (Silva, 2001a, p. 400)
}

La ciudad y, por ende, el espacio público, en tanto espacio, no solo refiere a aspectos topológicos sino también a espacios virtuales. Tal término alude a las cosas, mundos y fenómenos que se perciben como reales pero que carecen de una sustancia física tangible; un espacio virtual puede no tener equivalente en el mundo real, pero la persuasión de su representación nos permite responder a este como si se tratase de uno real (Laurel, Strickland y Tow, 1994). La apropiación de estos espacios virtuales por parte de los colectivos sociales, en su acepción positiva, significa la extensión de los límites del propio concepto, ya de por sí desdibujados, lo que permite que un mayor número de personas o internautas accedan, abstraigan, reconfiguren, apropien y legitimen este espacio y su simbolismo. Sin embargo, en su acepción negativa permite naturalizar procesos homogeneizantes y exacerba la inequidad, debido a las barreras que puede representar el que no cualquier ciudadano tiene acceso ni a los objetos tecnológicos que permiten la conexión virtual, ni al servicio de conexión a internet.

\section{Digitalización del espacio público y desmaterialización de la frontera como límite}

Volviendo a la idea de frontera, en su acepción de límite o borde, hay que recordar que sugiere un lugar tanto de diferenciación como de negociación. Se trata de un fulcro de interacciones asimétricas a veces polarizadas, particularmente 
desde la perspectiva sociopolítica, que se concibe en el relato cotidiano de quienes lo habitan, consumen o trascienden (Jorquera, 2011). Puede decirse que el valor simbólico asociado a un lugar corresponde a una dimensión psicosocial y puede ser independiente de una localización geográfica cuando su función principal es plasmar la memoria colectiva (Mercado y Hernández, 2010).

La introducción del concepto de liminalidad facilita la comprensión de la dinámica entre la forma espacial de la frontera y su función como borde espacial. Caracteriza tanto al espacio que resulta del tránsito o desplazamiento como a la transición de un ambiente a otro, a través de un límite. No se refiere exclusivamente a su dimensión material o morfológica: el espacio liminal considera también la dimensión psicosocial donde se gestionan relaciones dentro de una estructura social (Fourny, 2013).

La red, internet y la virtualidad coadyuvan a la desmaterialización de la frontera como límite y permiten la celebración de otro tipo de apropiación del espacio público en su dimensión simbólica y física. Incluso se habla de una nueva estructura social llamada a ser la ciudadanía del universo de las redes, igualmente basada en la comunicación e interacción entre personas que expresan e institucionalizan modos de ver la realidad.
... en la Red hay también espacios que inducen al diálogo, promueven la interacción e incluso, de manera explícita, abordan, documentan y enriquecen la reflexión sobre temas de la mayor relevancia para nuestras sociedades. Allí hay estruendoso espacio público pero también, junto con él, hay sitio para un ejercicio enterado y racional, que acaso sea capaz de articular la esfera pública [... y que] ha transformado los parámetros espaciales, los horizontes persona- les, la concepción que tienen de su entorno y del mundo, así como las capacidades para socia- lizar de centenares de millones de usuarios de Internet. Para muchos de esos internautas la Red es hoy parte de sus experiencias cotidianas y en ella disponen de nuevas opciones para entablar, expandir y/o diversificar sus vínculos sociales. (Trejo, 2009, p. 8)

Con el desarrollo tecnológico se espera que cada vez más personas converjan en el espacio público virtual y más actividades se lleven a cabo en él. Coca et al. (2015) tocan el tema de lo irreal de los imaginarios, no como una argumentación de su inexistencia o invalidez, sino como el reconocimiento de otro plano de existencia, y establecen un análisis del aporte teórico de Xavier Zubiri, quien identifica tres modos de materialización de lo irreal: el espectro, relacionado con la ilusión y, por otro lado, la ficción y la idea mayormente ligadas al tema del imaginario. (Zubiri, 1999)
Zubiri ha mostrado en sus primeros escritos como la virtualidad es un elemento fundamental que caracteriza al 'ser intencional', de ahí que este no sea realidad, sino -efectivamente- virtualidad [...] la irrealidad es el proyecto de una realidad. De ahí que la irrealidad es cuasi-creación [...] Por esto, la irrealidad juega un papel fundamental dentro de los imaginarios sociales ya que son ese conjunto de esquemas [...] que más que permitirnos percibir, explicar e intervenir lo que hacen es configurar un marco utópico (sin lugar) $y$, por tanto, futurible que nos conducen por una determinada senda de creación de nuestro entorno. (Coca et al., 2015, p. 119

Es decir, el desarrollo tecnológico, la opción a la creación de redes virtuales a través del internet y, sobre todo, dispositivos móviles, viabilizan nuevos y más eficientes métodos de comunicación que devienen en nuevas estructuras ciudadanas que a su vez se apropian de este nuevo espacio público, que además prefigura en el imaginario gestado la senda que determina la realidad futura, con base en la irrealidad presente e institucionalizada.

\section{El caso de Ciudad Juárez (México)}

La frontera norte de México (Figura 1) se conforma de 3185 kilómetros, cuenta con 11 ciudades pares o "ciudades espejo", que es un concepto geográfico que según Reyes (2001) (citado en García, 2007) es aplicado a localidades urbanas contiguas las cuales se encuentran separadas por un límite administrativo nacional o internacional, donde las interrelaciones económicas, políticas y sociales presentan un grado de complementariedad. Como caso de estudio se plantea hacer un análisis del imaginario colectivo dentro del borde de frontera como un espacio público binacional entre las ciudades fronterizas de Ciudad Juárez y El Paso, Texas.

Para tener una mejor comprensión de la dinámica espacial entre estas dos ciudades, se indaga el proceso histórico de su conformación. En el año 1659, el fraile franciscano fray García de San Francisco fundó lo que hoy son Ciudad Juárez y El Paso con el nombre de Misión de nuestra Señora de Guadalupe de Mansos del Paso del Río del Norte; esta misión fue un paso obligado para el

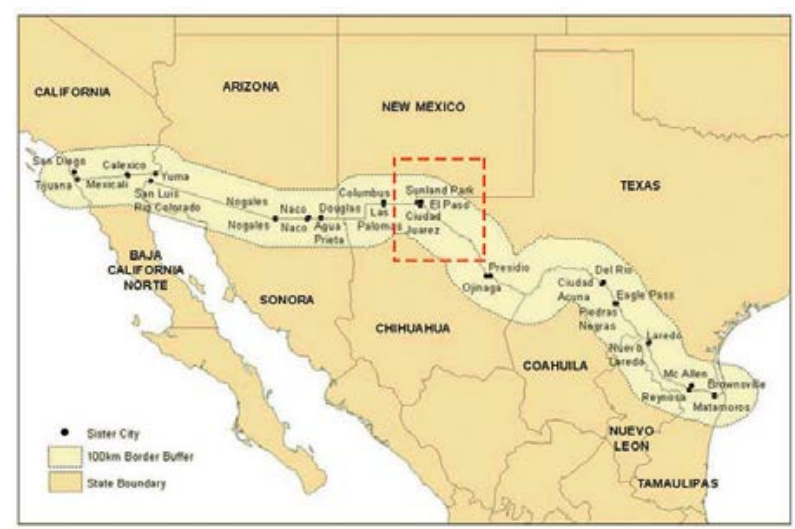


camino que unía al centro de México con la ciudad de Santa Fe, y uno de los pocos puntos donde se podía cruzar el caudaloso río Bravo (Rayas, 2016). Para el año de 1848, como resultado del Tratado de Guadalupe-Hidalgo, México vendió a Estados Unidos más de 2 millones de kilómetros cuadrados de territorio que corresponden a lo que actualmente son California, Nuevo México y Texas. El río Bravo quedó entonces como el límite entre las dos naciones. En el año de 1963, con la devolución del área conocida como el Chamizal por parte de Estados Unidos al territorio mexicano, este límite se ha mantenido hasta su actualidad (Sepúlveda, 1958) (Figura 2).

Actualmente, esta frontera es la segunda en importancia por debajo de Tijuana-San Diego, cuenta con 2,4 kilómetros de frontera delimitada por el río Bravo. Ciudad Juárez tiene una población de 1'508.044 habitantes y la población de El Paso es de 683.080 habitantes, con un área binacional con más de 2 millones de habitantes con una extensión de 984,9 km². Esta área puede ser definida como una metrópolis transfronteriza; este concepto, según Alegría (2009), se puede definir como una unidad urbana binacional donde existe una urbanización continua y una relación intensa entre las dos unidades urbanas, aunque también se puede definir como un espacio de carácter binacional.

\section{El espacio público fronterizo}

Los espacios públicos son el referente de mayor importancia al momento de analizar la relación entre la ciudad y el habitante. Como lo menciona De las Rivas (1992), el espacio tiene su propia identidad, y el hombre, al moverse dentro de este, lo lleva y lo modifica según su propia percepción. Se entiende como espacio público todo aquel lugar de libre tránsito como lo son las calles, los parques y las plazas, donde los individuos interactúan entre sí y forman lazos que fortalecen el tejido social.

El espacio público es, entonces, el generador de la identidad, y se va modificando conforme a las aspiraciones del usuario. El espacio público se puede producir de dos maneras: artificial y natural.

La producción artificial se refiere al espacio creado por urbanistas, arquitectos y gestores de la ciudad, estos espacios se diseñan mediante valores estéticos y de uso, retomando elementos técnicos y academicistas. Por otro lado, el espacio público natural es el creado por los mismos ciudadanos al apropiarse de él, asignándole significados, valores subjetivos y estructura identitaria; genera un gran sentido de apropiación y arraigo por parte del usuario, ya que este espacio le es más representativo que los de carácter artificial, dado que, en primera instancia, no ha participado en su construcción, no ha invertido tiempo y no le produce evocaciones emocionales.

Si bien prácticamente todas las ciudades fronterizas tienen sus propias particularidades que las hacen dignas de valiosos análisis, Ciudad Juárez cuenta con los dos tipos de producción de espacio público, donde resaltan aquellos que brindan un lugar de encuentro, descanso, socialización, recreación a los habitantes y participación en la actividad on line.

A manera de contextualización, se tiene que los espacios públicos tradicionales más representativos están ubicados dentro del primer cuadro de la ciudad, el centro histórico. La importancia del centro histórico como un espacio público de relevancia está dada por su proximidad a uno de los cruces fronterizos con El Paso. Una de las particularidades de este espacio es que ambos centros históricos se encuentran conectados por un mismo eje, lo que hace que el flujo sea continuo y sin límites evidentes.

Para los habitantes de Ciudad Juárez, dentro de los espacios públicos tradicionales que consideran de mayor importancia se encuentran El Chamizal (Figura 3), un parque público federal ubicado justo en la frontera, que a su vez es el único territorio que ha sido devuelto por Estados Unidos; el centro histórico y los centros comerciales en conjunto.

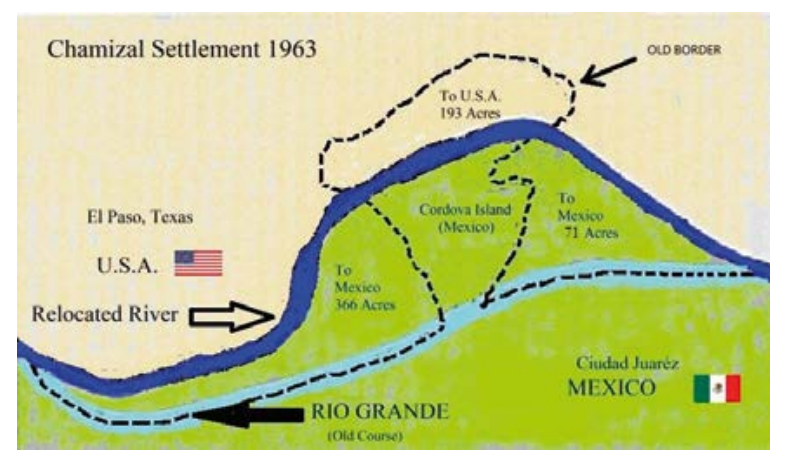

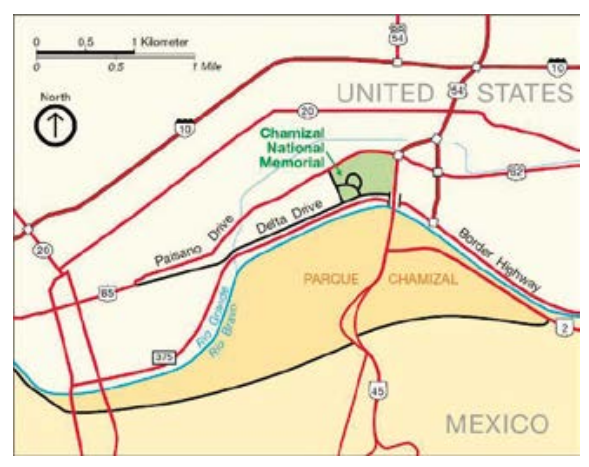

4 Figura 2. Mapa de El Chamizal en 1963 Fuente: Hayes (2010)

(3) Figura 3. Parque público El Chamizal Fuente: Archambeau (2017). 
El centro histórico de Ciudad Juárez está localizado al margen del río Bravo y próximo al Puente Internacional Paso del Norte, es un espacio con gran simbolismo dentro de la ciudad; su uso habitacional, comercial y de servicios, acompañado de una infraestructura adecuada, lo convierten en el corazón comercial, histórico-cultural, religioso y administrativo. Este es uno de los espacios de la ciudad que propicia la generación de un imaginario colectivo: por una parte, el sentimiento de nostalgia en cuanto a lo que un centro histórico representa para los ciudadanos, por otra, es el referente de identidad en la ciudad, lo que representa en el aspecto físico y se refleja socialmente. Es por eso por lo que este espacio de carácter tradicional es tan importante para los habitantes fronterizos.

Aunado a una inusual ola de violencia, consecuencia de la guerra contra el narcotráfico, Ciudad Juárez fue considerada durante el periodo 2006-2013 como la ciudad más violenta del mundo, según estadísticas del Consejo Ciudadano de Seguridad Pública y la Justicia Penal (CCSPJP, 2011), con una tasa de 229 homicidios por cada 100 mil habitantes; incluso por encima de lugares en conflicto bélico como Kandahar, Afganistán, con 169; San Pedro Sula, Honduras, con 125; Caracas, Venezuela, con 118 homicidios. En el quinto lugar de la clasificación está la capital de Chihuahua, con una tasa de 113 homicidios por cada 100 mil habitantes, seguida por el Distrito Central; Tegucigalpa, Honduras, con 109 y la capital de Guatemala con 106.

El centro histórico fue alguna vez la ciudad toda y, por tanto, síntesis de la diversidad que lo caracteriza. Por lo general, las ciudades tienen en su centro histórico el origen de su propia vida, y así como se desarrollaron con el transcurso de los años, hoy deben retomar sus pasos desde estas raíces; el centro es el origen y el fin mismo de la ciudad (Carrión, 2005). Aunque con el crecimiento de las urbes se originen nuevos centros o centralidades, el centro histórico jamás dejará de tener importancia ya que este espacio emblemático queda en la memoria de los ciudadanos.

A pesar de los problemas que enfrenta -deserción, violencia y falta de apoyo por parte de las autoridades correspondientes- sigue siendo el espacio público por excelencia, es aquí donde están las plazas más importantes de la ciudad, la catedral, los museos, las escuelas de arte, los comercios de distintos tipos; esto le da una gran relevancia, ya que suele ser el ancla para los turistas y compradores extranjeros, lo que convierte a este lugar en un espacio multifuncional donde se puede percibir la realidad de la sociedad juarense, que se encuentra ligada al borde, tanto en la esfera de lo simbólico, como en su ubicación geográfica contigua.

\section{Metodología}

El estudio contempla un enfoque metodológico de corte cualitativo y alineado a lo que establecen Castoriadis (2004) y Silva (2006). Aunque el análisis de los imaginarios tiene un carácter y escala netamente social, estos se manifiestan a través del individuo, es decir, cada persona es la resonancia del contexto al que pertenece. Castoriadis (2004) establece que no existe una metodología exacta y precisa, en tanto no puede hablarse de un único imaginario, acotado, exacto y preciso para el grupo social analizado. Con ello, tanto el proceso de recolección de información, como la calidad del material establecen la lógica de interpretación.

Se retoma lo establecido por Silva (2006) al respecto de la encuesta, la entrevista y los registros audiovisuales, como herramientas que hacen operable el análisis de los imaginarios. En una primera parte, se diseñó un instrumento que constó de tres preguntas de identificación del sujeto (edad, sexo y ocupación) para poder observar si existen tendencias de acuerdo con estas variables; seis preguntas abiertas, para dar el espacio a la generación de discurso libre por parte del individuo, en tanto se reconoce que a través de la expresión libre del lenguaje dicho individuo no solo comunica ideas, sino que se somete a un proceso de pensamiento retrospectivo en el que se observa la construcción que hace de su propia realidad (Austin, 1982), y, por último, ocho preguntas estructuradas bajo la escala Likert, que de acuerdo con Matas (2018): "son instrumentos psicométricos donde el encuestado debe indicar su acuerdo o desacuerdo sobre una afirmación, ítem o reactivo, lo que se realiza a través de una escala ordenada y unidimensional" (p. 39), que en este caso se enfocaron en ítems ligados a la temática del borde fronterizo y su presencia en la cotidianidad del encuestado, como espacio de apropiación física y virtual, así como el uso de plataformas digitales para denotar su presencia.

En este punto, una vez establecido el análisis del imaginario del espacio público físico en la frontera, se realizó una aproximación al análisis de la apropiación de dicho espacio en su calidad de virtual, o irreal (enunciado por Zubiri, 1999) y su relación con la construcción de las ideas y las ficciones que lo materializan y establecen las sendas de la ciudad, del espacio público futurible.

En esta etapa se tuvo la participación de 32 habitantes de ambos lados de la frontera, entre 19 y 59 años (43,8\% mujeres y $56,3 \%$ hombres), 
con áreas de ocupación diversa a fin de no sesgar la información por un tipo de filtro particular para entender el mundo, por lo que se distribuye en especialistas del área de ciencias de la salud, ciencias exactas, humanidades, oficios varios y estudiantes. Se realizaron 10 preguntas abiertas, 3 de opción múltiple y 7 preguntas de escala tipo Likert.

En una segunda parte, se realizó un análisis de los registros mediáticos disponibles, en tanto estos constituyen uno de los principales recursos para la consolidación de imaginarios y representaciones. Dado que los imaginarios no se forman de manera aislada por eventos particulares, es necesario considerar más de uno para su análisis, con la intención de detectar los patrones o puntos de coincidencia que den luz sobre los mencionados imaginarios de la frontera. Por lo anterior, los eventos seleccionados responden a temáticas variadas respecto a actividades significativas que se han generado en el borde fronterizo, en tanto evidencias de apropiación:

- Chupacabras $100 \mathrm{~km}^{2}$ : evento deportivo de ciclismo de montaña.

- Hugs not walls ${ }^{3}$ : evento organizado por la Red en Defensa de los Derechos de los Migrantes, que permite la reunión física, por unos minutos, de familiares divididos entre México y Estados Unidos, por su situación migratoria.

- Misa binacional: Celebrada en el marco de la visita del papa Francisco a México.

- Jane's Walk ${ }^{4}$ : evento cultural y de integración social que busca urbes a "escala humana" realizando recorridos por la ciudad.

- Letras a la border ${ }^{5}$ organizado por Nómada Laboratorio Urbano.

- Protestas/manifestaciones sociales: eventos cubiertos por los medios locales.

Asimismo, se tomó como referencia y para el registro visual de la ciudad el trabajo del fotógrafo David Alvarado ${ }^{6}$, quien publicó en la plataforma de la revista Vogue México ${ }^{7}$ un documental que lleva por título 'Bordertown': el otro rostro de Cd. Juárez. David Alvarado retrata la cotidianidad de la ciudad fronteriza (2017, 23 de febrero).

\footnotetext{
http://chupacabras100kms.org/

http://bnhr.org/hugs-not-walls-stories-of-family-reunification/

4 https://janeswalk.org/mexico/ciudad-juarez/

https://www.nmdlab.com/Letras-a-La-Border

6 Fotógrafo mexicano-estadounidense, nacido en Santa Ana, California.

7 http://www.vogue.mx/agenda/cultura/articulos/ proyecto-bordertown-del-fotografo-david-alvarado/6988
}

\section{Resultados}

\section{El borde fronterizo como espacio público binacional}

No solo los espacios públicos tradicionales permanecen en el imaginario colectivo; así como se mencionó, existen dos tipos de origen de los espacios públicos, el artificial y el natural. El espacio natural se considera un lugar donde, a pesar de no cumplir con las características que se espera, debe tener un espacio público, este funciona como tal, es este espacio el resultado de la apropiación de la misma sociedad que lo dota de una gran carga de identidad y significado.

En el caso de Ciudad Juárez se puede decir que este espacio público natural es el borde fronterizo. El borde es el límite entre las dos ciudades y se encuentra delimitado por el río Bravo. El término borde refiere al área inmediata en ambos lados de la frontera. Esta línea fronteriza cuenta con aproximadamente $50 \mathrm{~km}$ de línea y con 4 puentes internacionales localizados en distintos puntos de la ciudad (Figura 3). En el desarrollo de la mancha urbana en ambos lados de la frontera se puede apreciar que las ciudades tienden a crecer al margen de la línea, tratando de abarcar lo más posible el lecho fronterizo, lo que genera una traza un tanto lineal.

Los espacios más representativos se localizan lo más cercano posible a este borde, los centros históricos de Juárez y el El paso se encuentran a escasos metros de esta línea, así como infraestructura urbana como las unidades administrativas de ambas ciudades, teatros, plazas, hospitales, escuelas, parques y parques industriales. Esta línea, aunque cuenta con límites físicos como el denominado muro fronterizo, se encuentra interrumpida por la interacción de los puentes fronterizos, que pueden ser entendidos como puntos de hibridación espacial donde el límite territorial es intangible (Figura 4).

(v) Figura 4. Bridge of the Americas POE, El Paso, Texas Fuente: Tourtellotte (2016).

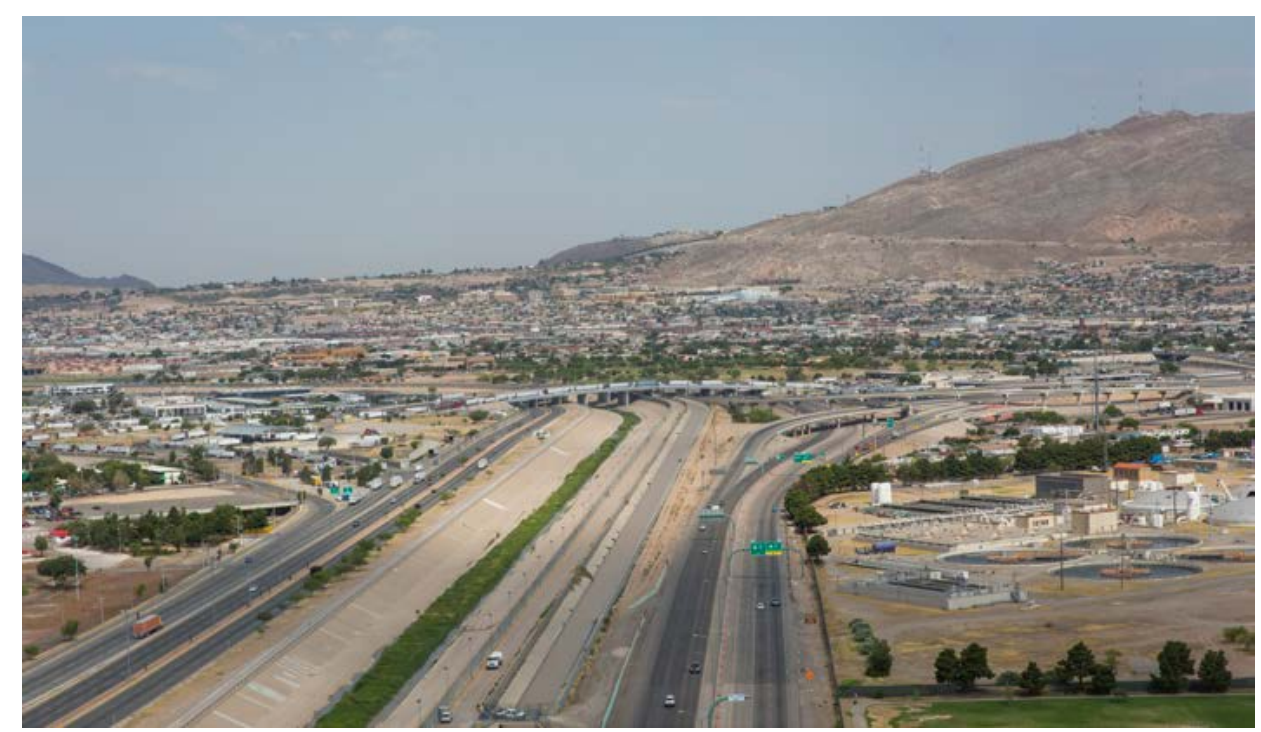


Estos puntos de conexión entre ambas ciudades generan un flujo continuo de tránsito, que el habitante fronterizo conceptualiza como uno mismo, ya que las relaciones continuas invisibilizan este límite para la percepción de los fronterizos; las relaciones sociales, comerciales y culturales son permanentes y continuas. Estos nodos de hibridación pueden ser considerados un espacio de carácter binacional. Este espacio en particular carece de muros u obstáculos que impidan circular de un lado a otro justo por encina de la línea fronteriza. Así, en el espacio de los puentes existe desde actividad comercial (venta de divisas, artesanías, alimentos y bebidas), hasta de protesta social (manifestaciones).

Así como los puentes internacionales son nodos de hibridación, el borde a lo largo de la frontera puede ser visto como un espacio de interacción. Paradójicamente, aunque el borde no se encuentre considerado como un espacio público, la apropiación de la sociedad en ambos lados de la frontera lo ha ido transformando y le ha dado un cierto índice de importancia para el imaginario colectivo fronterizo. De manera paralela, los puentes tienen ya una presencia como espacio público virtual, en tanto son monitoreados constantemente, y en tiempo real se tiene el reporte del escenario previsto para el cruce en medios de transporte motorizados ${ }^{8}$.

Dentro de las representaciones de apropiación que se pueden percibir en este espacio público se encuentran las siguientes actividades de carácter social, político, cultural y deportivo. En el aspecto social, los cruces internacionales son los escenarios predilectos para realizar manifestaciones de carácter político y social, ya que al interrumpir el flujo en los puentes internacionales los manifestantes reciben un mayor nivel de atención por parte de las autoridades para escuchar sus reclamos y poder llegar rápidamente a una solución que beneficie a ambas partes. Así, este espacio se ha convertido en el referente para manifestaciones de toda índole.

Algunos ejemplos de estas dinámicas binacionales se encuentran en el proyecto Hugs not Walls ${ }^{9}$ donde, con la autorización y bajo la supervisión de las autoridades, se permite la apertura de la frontera para que familias que se encuentran separadas por problemas migratorios puedan convivir en este espacio intermedio, el borde del río Bravo. Esta es una de las iniciativas por parte del Gobierno para poder incentivar la creación de estos espacios públicos binacionales.

En el aspecto cultural, el borde del río Bravo es el escenario de constantes manifestaciones

8 Al puente: https://alpuente.mx/juarez/

9 http://bnhr.org/hugs-not-walls-stories-of-family-reunification/ culturales como murales y pintas con la intención de manifestar inquietudes de carácter político. Existen actualmente diversos colectivos de arte que se empeñan en convertir este borde en un espacio de expresión de la cultura fronteriza. Este tipo de iniciativas que se manifiestan en ambos lados de la frontera tienen buena aceptación por parte de la población fronteriza. Un ejemplo es el proyecto Jane's Walk ${ }^{10}$, el cual es un movimiento global de caminatas inspiradas en la urbanista Jane Jacobs, para construir ciudades basadas en la comunidad. Cada año, el primer fin de semana de mayo se realiza un festival de caminatas en más de 200 ciudades del mundo con la finalidad de que la sociedad conozca su ciudad, la viva y la comprenda.

Otro de los movimientos socioculturales que se puede percibir como resultado de dinámicas y participación social binacional más significativas es el movimiento \#LetrasALaBorder, iniciativa de Nómada Laboratorio Urbano ${ }^{11}$, donde por medio de un proyecto-intervención binacional se hizo visible el imaginario de las y los habitantes de la frontera, a través de un consenso ciudadano. Este proyecto tiene como objetivo resaltar la multiculturalidad y el lazo binacional de la frontera Juárez-El Paso a través de la instalación de un letrero urbano, en el límite geográfico de la frontera, en el que se depositan frases y pensamientos propositivos generados por la propia ciudadanía (Figura 5). Como parte de la metodología de este interesante proyecto se llevó a cabo un ciclo de talleres participativos con ciudadanos de ambos lados de la frontera.

De igual manera, cabe mencionar el Foro de diálogo cultural transfronterizo, el cual tiene como objetivo abrir canales y puentes de discusión entre las fronteras utilizando el lenguaje del arte, periodismo, investigación, cine, música, antropología social, urbanismo, entre otros, con el apoyo directo de la comunidad para intercambiar ideas. El objetivo central es reflexionar sobre los procesos y fenómenos que atraviesan las fronteras en el mundo, donde se puedan identificar particularidades, entrelazar y construir nuevas formas donde sea posible desarrollarse como individuos y como sociedad. Este tipo de foros, programas y laboratorios urbanos tiene como finalidad interpretar, comprender y enaltecer el carácter fronterizo, sobre todo en una ciudad como Ciudad Juárez, la cual busca constantemente reinterpretar su identidad de frontera binacional con Estados Unidos.

Así como los movimientos ciudadanos que incentivan el carácter cultural de este espacio binacional, también los hay en el aspecto depor-

10 https://janeswalk.org

11 https://www.nmdlab.com/ 
tivo ya que el borde es el escenario de una de las carreras de bicicleta de montaña con mayor importancia en México, llamada el Chupacabras $100 \mathrm{~km}$, que cuenta con una participación aproximada de más de 2000 ciclistas y se realiza una vez al año. Esta carrera generó que, diariamente, el borde sea utilizado por ciclistas y corredores, y se ha convertido poco a poco en el espacio predilecto para la práctica de este deporte. Estos son algunos de los ejemplos más relevantes al momento de hablar y tratar de definir el borde fronterizo como un espacio público binacional.

\section{Discusión}

Entre lo que se rescata del discurso de los participantes, así como de las evidencias de los eventos analizados que tienen sede en el borde fronterizo, es que coinciden en otorgarle un carácter de espacio público apropiado, de gran carga simbólica e identitaria. En todos los eventos analizados se muestra, desde la perspectiva de quien los relata y de quien ha sido participante, una clara necesidad por hacer de dicho borde un lugar para denotar la fuerza de la presencia de los habitantes y su identidad, en tanto su estatus como fronterizos. Estos eventos, lejos enraizar los estigmas de la frontera como lugar de división y segregación, son manifiestos directos y abiertos de una comunidad que comparte ideales, intereses, lazos y preocupaciones.

Asimismo, el uso de dispositivos tecnológicos facilita dicha apropiación dado que, por un lado, permite la comunicación y el intercambio entre los habitantes de ambas fronteras, con lo que se propicia la desmaterialización de los muros físicos, y, por otro lado, permite la participación virtual en eventos transfronterizos, como en la misa binacional.

Siguiendo con este tópico, cabe mencionar que a pesar de que desde el sector académico se habla de la importancia y preocupación que suscita la privacidad y la seguridad en un entorno futuro dominado por la digitalización de las actividades humanas, resalta la postura tecnócrata del ciudadano común, al atribuirle al desarroIlo tecnológico en la frontera la posibilidad de desmontar, de manera virtual, algunos limitantes que implican el muro fronterizo, además de la resolución de problemas varios como: la provisión y accesibilidad de servicios (salud, transporte, vivienda, educación), el ahorro de recursos, la longevidad e ideas asociadas a la mejora en la calidad de vida y la comodidad.

El 31,3\% considera que el desarrollo de la tecnología "no representa ningún riesgo", mientras que el 12,5\% lo considera "absolutamente riesgoso", dejando una mayoría del 43,8\% en términos medios, con tendencia a la confianza, pues se le considera indispensable para vivir en la ciudad y ser partícipes de los eventos del borde. Un aspecto que denota la confianza en las posibilidades que ofrecen los dispositivos tecnológicos es que la mitad de los participantes reconocen que realizan movimientos bancarios binacionales cotidianos y los consideran confiables. Por su parte, el $62,5 \%$ considera que la tecnología es parte inherente de las actividades cotidianas y de intercambio en la frontera y que tendrá que avanzar aún más, con lo que parte de la preocupación es la constante necesidad de actualización del modelo de los teléfonos inteligentes. Un ejemplo puntual es que se menciona cómo antes de la digitalización de los principales negocios en Estados Unidos se requería hacer filas de horas para aprovechar ofertas, mientras que ahora pueden hacerse compras en línea en los mismos establecimientos, con lo que se refuerza la idea, entre los habitantes, de una sola comunidad transfronteriza en donde los muros divisorios desaparecen.

En el orden de la ubicación de la principal ventaja que representa el espacio público virtual sobresale la idea de la comunicación y resolución de problemas de manera más rápida y eficiente, lo que se alinea con la idea de la desmaterialización de la frontera como límite, incluso la deslocalización diferenciada y aparente de actividades domésticas y públicas. Respecto al orden de importancia de las actividades que los fronterizos señalan que les permiten apropiarse del espacio público virtual, en orden de importancia de mayor a menor están:

- Comunicación (redes sociales).

- Compra de productos varios.

- Movimientos bancarios.

- Viajes (compra de boletos de avión o de transporte terrestre).

- Pago de servicios.

- Transporte (Uber).

- Lectura.

- Juegos.
(7) Figura 5. "The border is a place not a limit"

Fuente: Nómada Laboratorio Urbano (2017).

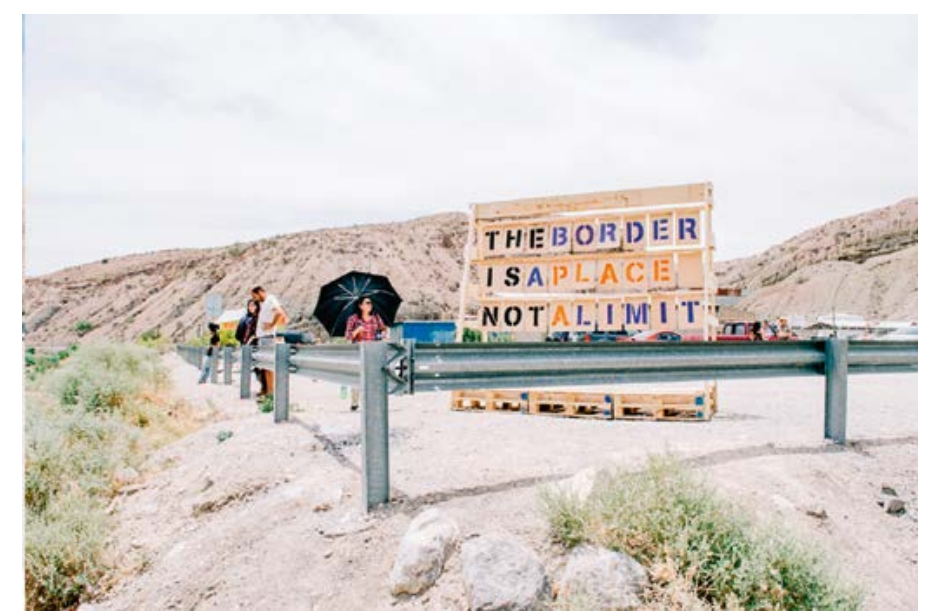


Como se observa, a pesar de que parte de los impactos negativos asociados al uso de plataformas digitales se vincula con la idea de la pérdida de ciudadanía, de valores sociales, de convivencia y, en general, el contacto entre personas, el uso que verifica la apropiación del espacio público virtual es precisamente la comunicación y el contacto con el otro.

De manera preliminar se observa que el imaginario del espacio público futurible de la frontera exalta la aspiración y el anhelo por la tecnología como salvaguarda de la integridad humana, y es esta misma la que genera esta virtualidad que expande los límites del espacio físico y borra la idea del borde como obstáculo. Aunado a esto, una de las principales ventajas que ven los ciudadanos en el uso de tecnologías de comunicación es la superación de ciertas anclas espacio-temporales, es decir, la posibilidad de hacer múltiples actividades a través de una conexión a internet, en un lugar y horario indistinto, con aseveraciones como: "puedo entregar mi tarea estando del otro lado" (de la frontera), "más tiempo para vivir este corto pero delicioso respiro" , "desde el hogar se pueden hacer muchas de las actividades que hoy se hacen en físico" o "cuando no es muy grave el pediatra de mi hijo que está del otro lado (de la frontera), me atiende por whats (WhatsApp)".

En el tema de los registros fotográficos resalta la vivencia simbólica retratada en lo cotidiano, enraizado en creencias y mixturas culturales. También sobresale el hecho contrastante entre la aproximación al imaginario a través del discurso de la entrevista, y la necesaria narrativa que cuenta la fotografía. Mientras que una se construye alrededor de la idea del progreso tecnológico, la modernidad y el continuo desarrollo, el trabajo fotográfico revela más bien un aire de nostalgia y la búsqueda desesperada por la exaltación de una identidad cultural. También es de notar que de las 38 fotos que componen la colección completa, solo en una es posible evidenciar la realidad tecnológica de la sociedad, y es debido a que se trata de una fotografía a contraluz que deja retratada la sombra de quien toma la cámara, a manera de imagen en negativo de la selfie, pues la persona permanece en el anonimato de su sombra y su silueta.

En la presentación de fotografías es casi inexistente la evidencia de la alta conectividad y la vida on line de un sector de la población en Ciudad Juárez. De hecho, el propio autor del material gráfico menciona que en la ciudad "aún se mantiene en el imaginario popular como agresiva. Muestra una cara desconocida de un lugar que creímos conocido", con lo que, de nuevo, su visión contrasta y perpetúa el estigma de la violencia.

Con ello puede reafirmarse lo que establece Castoriadis (2004) respecto a los imaginarios, y la falta de certeza para hablar de un solo imaginario verdadero para una sociedad, pues depende de quien lo interprete y su capacidad para identificar las instituciones instauradas, que darán pie a las interpretaciones y consideraciones. En este caso, mientras que el trabajo fotográfico que pretende describir la frontera está filtrado por los ojos añorantes del pasado, se observa que tanto en las encuestas como en las entrevistas y los registros de los medios locales se relata y se construye una realidad que da cuenta del borde fronterizo como espacio público apropiado, así como de la importancia de la tecnología y el mundo virtual que viabiliza, para desmontar la idea de la frontera como límite perpetuo e inamovible para los fronterizos. La tecnología vuelve el muro poroso, permeable, difuso y, en algunos casos, invisible.

\section{Conclusiones}

Es un hecho que las ciudades fronterizas son espacios complejos de acotar y abordar debido a su fenomenología como espacios transitorios, y multiculturales que, además, en su constancia como sistemas dan espacio para las contradicciones y los contrastes debido a su lógica inherente. Por un lado, son anhelo del pasado y la tradición y, por otro, la aspiración de la ficción de la tecnología como salvaguarda de la humanidad.

Sin embargo, por un lado, es posible identificar el espacio público anclado a territorios físicos muy específicos y de gran simbolismo para los fronterizos y, por otro, es evidente la inevitable transición a espacios deslocalizados geográficamente, espacios virtuales que se configuran como una nueva forma de espacio público que, entre otras cosas, permite la reconfiguración del imaginario del borde como límite y establece una nueva ciudadanía de redes que comparte, comunica, intercambia, socializa y, por tanto, legitima la apropiación de la ciudad a través de aparición en la red. Ya sea que se trate del análisis del espacio público físico o virtual, el imaginario del borde fronterizo, más que un obstáculo, se vive colectivamente como espacio extensivo a la ciudad y a los ciudadanos.

Este imaginario identificado, presente en los habitantes de la región analizada, da cuenta de la importancia y de la gran carga simbólica que representa el borde fronterizo como espacio para el encuentro, el intercambio y la perpetuación de la identidad colectiva, como una sola sociedad transfronteriza. Este imaginario construye una identidad 
alrededor del borde, la afianza y la despliega más allá del muro físico que divide territorial y legalmente los dos países, pues las relaciones sociales que se dan en el espacio físico, como estrategia de apropiación del espacio público, dan cuenta de la desmaterialización del borde como límite. Por el contrario, afianzado por el desarrollo tecnológico y por el surgimiento de una ciudadanía del universo de las redes, por un lado, podría hablarse de una frontera aumentada, $y$, por el otro, se permite confirmar la hipótesis respecto al surgimiento de un espacio público virtual que refuerza la desmaterialización simbólica del muro, que se traslapa con la existencia de un espacio público físico consolidado en el imaginario y las prácticas fronterizas.

\section{Referencias}

Alegría, T. (2009). Metrópolis transfronteriza. Revisión de la hipótesis y evidencias de Tijuana, México y San Diego, Estados Unidos. México: COLEF-Porrúa.

Alvarado, D. (2017, 23 de febrero) 'Bordertown': el otro rostro de Cd. Juárez. (Reseñado por Mariana Ramírez) Vogue México. Recuperado de http://www.vogue.mx/ agenda/cultura/articulos/proyecto-bordertown-del-fotografo-david-alvarado/6988

Archambeau, N. (2017, 26 de abril). El Chamizal map [Planimetría]. Recuperado de https:// history.colostate.edu/2017/04/history-students-use-digital-tools-explore-u-s-mexico-borderlands/area-map_2-el-chamizal/

Austin, J. (1982). Cómo hacer cosas con palabras. Barcelona: Paidós.

Brenna, J. (2011, julio-diciembre). La mitología fronteriza: Turner y la modernidad. Estudios Fronterizos, 12(24), 9-34. Recuperado de http://ref.uabc.mx/ojs/index.php/ref/ article/view/104/182

Carrión, F. (2005, agosto). El centro histórico como proyecto y objeto de deseo. Revista Eure, 31(93), 89-100. http://dx.doi. org/10.4067/S0250-71612005009300006

Carrión, F. (2016). El espacio público es una relación, no un espacio. En P. Ramírez Kuri (coord.). La reinvención del espacio público en la ciudad fragmentada. México: UNAM, Instituto de Investigaciones Sociales. Programa de Maestría y Doctorado en Urbanismo.

Castells, M. (2006). La era de la información: economía, sociedad y cultura (vol.1). La sociedad en Red. Madrid: Alianza Editorial.

Castoriadis, C. (1975). La institución imaginaria de la sociedad. Barcelona: Tusquets Editores.

Castoriadis, C. (2004). Sujeto y verdad en el mundo histórico social. México: Fondo de Cultura Económica.

CCSPJP (2011). Ciudad Juárez, por tercer año consecutivo, la urbe más violenta del planeta. México: Consejo Ciudadano para la Seguridad Pública y la Justicia Penal. Recuperado de https://www.seguridadjusticiaypaz.org.mx/sala-de-prensa/329-ciudad-juarez-por-tercer-ano-consecutivo-laurbe-mas-violenta-del-planeta

Coca, J., Valero Mataz, J., Randazzo, F. y Pintos J. L. (2015). Propuesta de una nueva conceptualización de los imaginarios sociales. En Lo imaginario: seis aproximaciones (pp. 113-128). Montreal: Tilde Editores.

De las Rivas, J. L. (1992). El espacio como lugar, sobre la naturaleza de la forma urbana. Valladolid: Universidad de Valladolid.
Environmental Protection Agency (2011). Map of the U.S. - Mexico Border Region [Planimetría] Dominio público. Recuperado de https://es.wikipedia.org/wiki/Archivo:Border_Region.png

Fourny, M. (2013). The border as liminal space. Journal of Alpine Research. Revue de géographie alpine, 101(2), 1-13. http://doi. org/10.4000/rga.2120

García Canclini, N. (2000). La ciudad espacial y la ciudad comunicacional: cambios culturales de México en los 90. En Rubens Bayardo (comp.). Globalización e identidad cultural. Buenos Aires: CICCUS.

García, M. (2007). Ciudades fronterizas del Norte de México. Anales de Geografía, 27(2), 41-57. Recuperado de https://revistas.ucm. es/index.php/AGUC/article/view/AGUC0707220041A

Hayes, M. (2010). Map of the Chamizal Dispute and Settlement of 1963 [Cartografía] Dominio público. Recuperado de https:// commons.wikimedia.org/wiki/File:Chamizal dispute map 01.jpg\#/media/Archivo:Chāmizal_dispute_map_01.jpg

Ito, T. (2006). Arquitectura de límites difusos. Barcelona: Gustavo Gili.

Jorquera, R. (2011). Ciudad del miedo: la seguridad y el capital social en las clases medias. Sonora: El Colegio de Sonora.

Laurel, B., Strickland, R. y Tow, R. (1994). Placeholder: Landscape and Narrative In Virtual Environments. ACM Computer Graphics Quaterly, 28(2). Recuperado de http://papers. cumincad.org/cgi-bin/works/Show?7475

Matas, A. (2018). Diseño del formato de escalas tipo Likert: un estado de la cuestión. Revista Electrónica de Investigación Educativa, 20(1), 38-47. http://doi.org/10.24320/ redie.2018.20.1.1347

Mercado Maldonado, A. y Hernández Oliva, A. V. (2010). El proceso de construcción de identidad colectiva. Convergencia. 17(53), 229251. Recuperado de http://www.scielo.org. mx/pdf/conver/v17n53/v17n53a10.pdf

Morales, A. y Cabrera, V. (2016). Imagen e identidad, unidad clave en el logro de la marca ciudad. Revista Legado de Arquitectura y Diseño, 11(20), 23-33. Recuperado de https://legadodearquitecturaydiseno.uaemex.mx/article/view/4758

Nómada Laboratorio Urbano (2017). [The border is a place not a limit. Fotografía] Recuperado de http://www.nmdlab.com/Letrasa-La-Border

Pineda, S. y Herrera, L. (2007, mayo-agosto). Ciudad Juárez: las sociedades de riesgo en la frontera norte de México. Fermentum. Revis- ta Venezolana de Sociología y Antropología, 17(49), 419-433. Recuperado de http://www. saber.ula.ve/handle/123456789/20770

Pintos, J. L. (1994). Sociocibernética: marco sistémico y esquema conceptual. En J. Gutiérrez y J. M. Delgado (eds.). Métodos y técnicas cualitativas de investigación social. Madrid: Síntesis.

Rayas, G. (2016, 1 de agosto). En 1659, el fraile franciscano Fray García de San Francisco fundó lo que hoy es tanto Ciudad Juárez como El Paso, Texas. Puente libre. mx. Recuperado de http://puentelibre.mx/ noticia/46502-en 1659 el fraile franciscano_/2

Reyes Posadas, C., Rangel Calvillo, E., Enríquez Denton, F. J. y Hernández Figueroa, E. (2001). Explorando la geografía de México 2. México: Editorial Nuevo México.

Sepúlveda, C. (1958). Historia y problemas de los límites de México: I La frontera norte. Historia Mexicana, 8(1), 1-34. Recuperado de https://historiamexicana.colmex.mx/ index.php/RHM/article/view/728

Silva, A. (2001a). Algunos imaginarios urbanos desde centros hi stóricos de América Latina. En F. Carrión (ed.). La ciudad construida. Urbanismo en América Latina (pp. $397-$ 407). Ecuador: Flacso.

Silva, A. (2001b). Imaginarios: estética urbana. En A. Vergara (coord.). Imaginarios: horizontes plurales. México: Instituto Nacional de Antropología e Historia, Escuela Nacional de Antropología e Historia.

Silva, A. (2006). Imaginarios urbanos: hacia el desarrollo de un urbanismo desde los ciudadanos. Bogotá: Convenio Andrés Bello, Universidad Nacional de Colombia.

Tourtellotte, J. (2016, 7 de junio). Bridge of the Americas POE. U.S. Customs and Border Protection El Paso Texas. [Fotografía] Dominio público. Recuperado de https:// commons.wikimedia.org/wiki/File:Bridge of the Americas POE El Paso Texas (27)793303644).jpg

Trejo, R. (2009). Internet como expresión y extensión del espacio público. MATRIZes, 2(2), 1-16. Recuperado de http://www.revistas. usp.br/matrizes/article/view/38225/40997

Zubiri, X. (1999). Primeros escritos (1921-1926). Madrid: Alianza/Fundación Xavier Zubiri. 



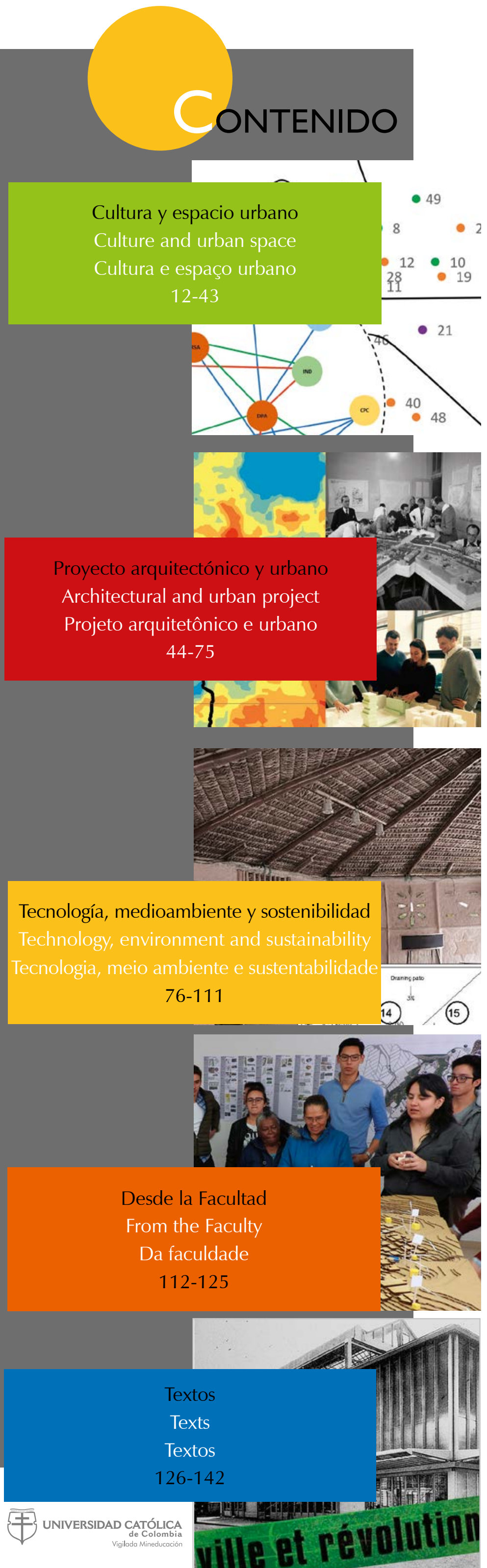

Arquitecturas colectivas y participación como estrategias para la construcción de la ciudad latinoamericana

Myriam Stella Díaz-Osorio

Pág. 3

ES

La caminabilidad en Bogotá: propósitos y condiciones socioespaciales que facilitan y limitan esta experiencia Pablo Páramo

Andrea Burbano

Pág. 12

ES EN

Planificación comunitaria en barrios socialmente

vulnerables. Identificación de los actores sociales en una comunidad

Rafael Alejandro Tavares-Martínez
Jesús Manuel Fitch-Osuna

ES

Pág. 22

Desvanecimiento de la frontera como límite. Imaginario del borde como espacio público físico y virtual

Gabriela Eloísa Muñoz-Torres

Susana Gutiérrez-Luna

Pág. 33

Estudiantes latinoamericanos en el Institut

d'Urbanisme de l'Université de Paris (1923-1941)

$$
\begin{aligned}
& \text { Andrés Ávila-Gómez } \quad \text { Pág. } 44 \\
& \text { ES }
\end{aligned}
$$

Apuntes para el repensamiento de la enseñanza de la Arquitectura. La cuestión epistemológica y la necesidad de una razón ampliada
Juan J. Álvarez-Álvarez
Pág. 57

ES

Equipamientos colectivos: "lugares" de producción de capital social
José Mario Mayorga-Henao
Pág. 68

\section{ES}

Bucle multidisciplinar para la sustentabilidad urbana

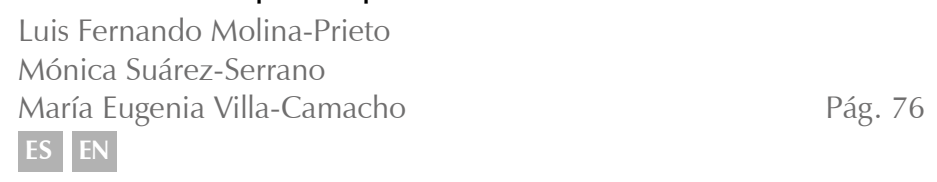

Durabilidad de los materiales naturales de construcción: percepciones de proyectistas, constructores y usuarios en Florianópolis, Brasil

Andrea Salomé Jaramillo-Benavides
Zuleica Maria Patricio-Karnopp
Lisiane Ilha-Librelotto

Pág. 89

\section{ES}

Thermal comfort in buildings for wet processing of coffee

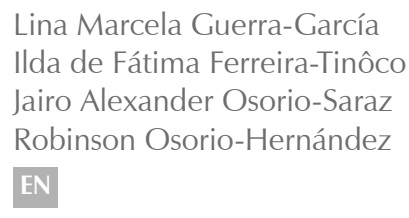

La arquitectura en los barrios: puntos de encuentro entre la academia y el saber popular

Hernando Carvajalino-Bayona

Pág. 112 ES

Arquitectura, modernidad, modernización

$$
\begin{aligned}
& \text { Jean-Louis Cohen } \\
& \text { Traductores } \\
& \text { Andrés Avila-Gómez. } 126 \\
& \text { Diana Carolina Ruiz } \\
& \text { ES }
\end{aligned}
$$


Arquitecturas colectivas y participación como estrategias para la

Arquiteturas coletivas e participação como estratégias para construir a cidad latino-americana

\section{Stella Díaz-Osorio}

La caminabilidad en Bogotá: propósitos y condiciones socioespaciales que facilitan y limitan esta experiencia

The Walkability of Bogotá: purposes and socio-spatial conditions that facilitate and limit this experience

A caminhabilidade em Bogotá: propósitos e condições socioespaciais que facilitam e limitam essa experiência

\section{Andrea Burbano}

Planificación comunitaria en barrios socialmente vulnerables.

Identificación de los actores sociales en una comunidad

Community planning in socially vulnerable neighborhoods.

Identification of social actors in a community

Planejamento comunitário em bairros socialmente vulneráveis.

Identificação dos atores sociais em uma comunidade

Rafael Alejandro Tavares-Martínez

esús Manuel Fitch-Osuna

Desvanecimiento de la frontera como límite. Imaginario del borde como espacio público físico y virtual

$m$

迹

Durabilidad de los materiales naturales de construcción: percepciones de proyectistas, constructores y usuarios en Florianópolis, Brasil

Durability of natural building materials: Perceptions of designers, builders, and users in Florianópolis, Brazil

Durabilidade dos materiais naturais de construção: percepções de projetistas, construtores e usuários em Florianópolis, Brasil

\section{Zuleica Maria Patricio-Karnopp}

Lisiane Ilha-Librelotto

Thermal comfort in buildings for wet processing of coffee

\section{Confort térmico en edificaciones para procesamiento húmedo de café}

Conforto térmico em instalaçōes para processamento úmido de café

\section{Ida de Fátima Ferve-Tartióco}

airo Alexander Osorio-Saraz

Robinson Osorio-Hernandez

La arquitectura en los barrios: puntos de encuentro entre la academia y el saber popular

Architecture in neighborhoods: meeting points between academia and popular knowledge

A arquitetura nos bairros: pontos de encontro entre a academia e o saber popular Hernando Carvajalino-Bayona

\section{Arquitectura, modernidad, modernización}

Architecture, modernity, modernization

Arquitetura, modernidade, modernização
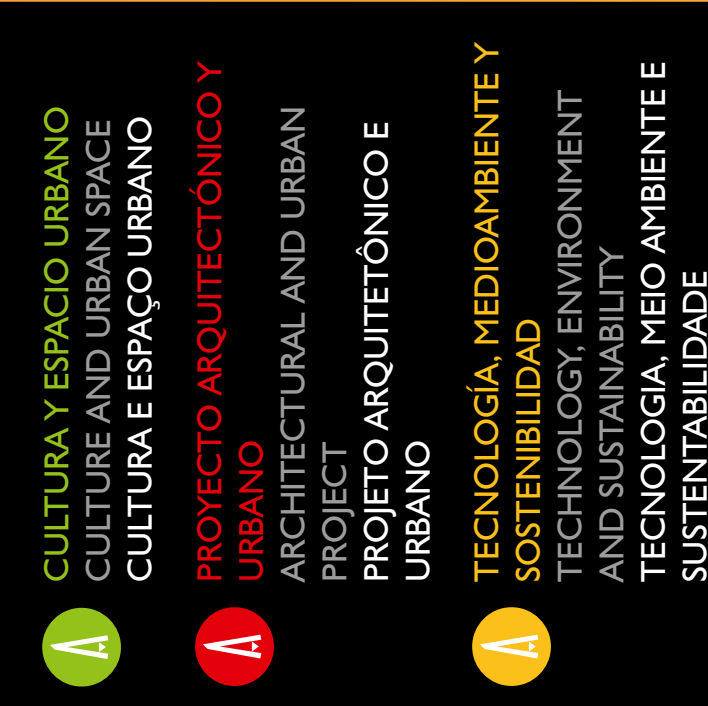

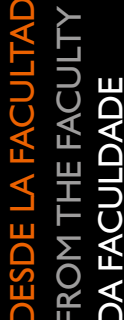

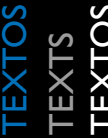
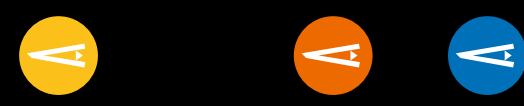

1) Clarivate

publind

Clarivate
Analytics

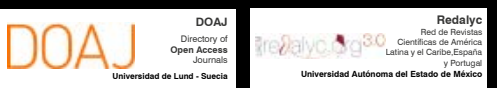

n.

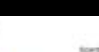

REDIB

EBSCO

cis

latindex unmoxom

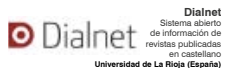

LatinREV

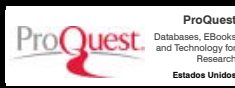

MIคR

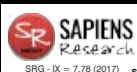

Goosin

Google

ARLA

hi. https://www.mendeley.com/profles/revista-de-arquitectura-bogot/

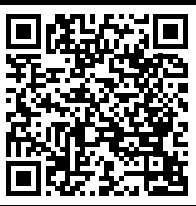

ISSN: 1657-0308 